\title{
Investigation of Laser-induced Welding Between Gold and Silver Nanostructures by Advanced TEM Techniques
}

\author{
Xiaohui $\mathrm{Xu}^{1}$, Subhajit Kundu ${ }^{1}$, Tugba Isik ${ }^{2}$ and Volkan Ortalan ${ }^{1}$ \\ 1. School of Materials Engineering, Purdue University, West Lafayette, USA. \\ 2. School of Mechanical Engineering, Purdue University, West Lafayette, USA.
}

Plasmonic nanostructures, such as gold and silver nanoparticles (NPs) or nanowires (NWs), are featured by their localized surface plasmon resonance (LSPR) under specific electromagnetic wavelengths. The LSPR behavior of plasmonic nanostructures can be modified either by changing the composition, shape and size or by nanojoining [1-3]. Specifically, laser has been proved to be an effective tool to join gold or silver nanostructures resulting in more flexible nano-blocks that can be applied to plasmonic devices, electronics, cancer therapy, etc. [4-6]. Although laser-induced welding of plasmonic nanostructures has been studied extensively [6-10], few studies focused on the welding between different species, for example, gold and silver. However, welding between gold and silver might provide a novel way to produce nanostructures with unique plasmonic or optical properties. On the other hand, most studies on laser-induced welding are ex situ, due to the difficulty of observing laser-material reactions at nanoscale. This has prevented us from gaining more details of the welding process.

With the advent and development of ultrafast TEM (UTEM), in situ study of laser-induced dynamic processes becomes feasible [11,12]. In this work, we conducted an in situ investigation of the welding between gold and silver nanostructures by using UTEM. Figure 1 shows the UTEM configuration we adopted for this work. Electrons were generated via a thermionic gun to form high-resolution images. A pulsed green laser, with a wavelength of $532 \mathrm{~nm}$ was utilized to initiate the welding process. An electronic delay generator was used to control the number of laser pulses arrived at the sample which is composed of both Au and Ag nanostructures, such as Au NP + Ag NP, Au NP + Ag NW, etc. The structural evolution was monitored via TEM images taken after different numbers of laser pulses.

The composition of Au-Ag nanostructures after UTEM experiments was studied by scanning transmission electron microscopy (STEM) and energy-dispersive X-ray spectroscopy (EDX). Figure 2 shows the STEM images and EDX results of Ag-Au NP pairs as an example. Four primary configurations of composition were revealed, with an unquestionable time sequence among them, which indicates the compositional evolution of Au-Ag NPs during welding. Based on this, we divided the welding process into five stages: in the first stage, a neck was formed between Au and Ag NP but no inter-diffusion (Figure 2a); in the second stage, a Ag layer formed above the Au NP, resulting in a $\mathrm{Au} @$ Ag core-shell structure (Figure 2b); in the third stage, the core-shell NP mentioned above transformed into an alloy, while a Au layer was formed around the Ag NP on the other side (Figure 2c); in the fourth stage, the Ag@Au core-shell structure formed in the third stage also became a uniform alloy (Figure 2d). We believe that inter-diffusion and surface diffusion will continue with further laser irradiation until two alloy NPs merge into one with homogeneous composition, which marks the final stage of welding. In this presentation, TEM images and more EDX results showing the structure evolution of Au-Ag NPs and a complete welding mechanism will be discussed. Furthermore, multiparticle welding and welding between different types of plasmonic nanostructures, including gold/silver NWs and NPs, will also be discussed. The interesting structures formed during Au-Ag nano-welding might inspire the fabrication of novel plasmonic devices. 
References:

[1] K.L. Kelly et al, J. Phys. Chem. B 107 (2003) p. 668.

[2] C. Graf et al, Langmuir 19 (2003) p. 6693.

[3] Y. Ma et al, RSC Adv. 6 (2016) p. 75916.

[4] H. Huang et al, J. Appl. Phys. 112 (2012).

[5] G. Baffou, R. Quidant, Laser Photonics Rev. 7 (2013) p. 171.

[6] A. Hu et al, J. Laser Appl. 24 (2012) p. 42001.

[7] Z. Peng et al, J. Phys. Chem. B 110 (2006) p. 2549.

[8] T. Zhang et al, Open Surf. Sci. J. 3 (2011) p. 76.

[9] S.J. Kim, D.J. Jang, Appl. Phys. Lett. 86 (2005) p. 1.

10] E.C. Garnett et al, Nat. Mater. 11 (2012) p. 241.

[10] V.A. Lobastov, R. Srinivasan and A.H. Zewail, Proc. Natl. Acad. Sci. U. S. A. 102 (2005) p. 7069.

[11] D. Shorokhov, A.H. Zewail, J. Chem. Phys. 144 (2016) p. 80901.

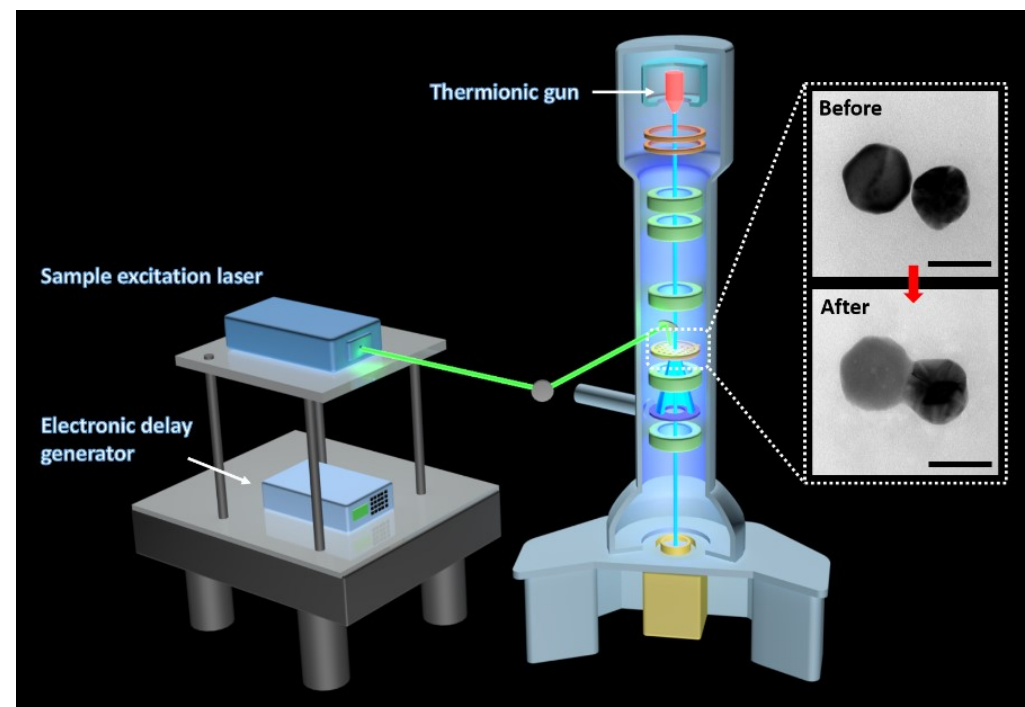

Figure 1. Schematic of 200kV Ultrafast TEM. Inset: two nanoparticles (Au and Ag) before and after laser irradiation. Scale bar is $50 \mathrm{~nm}$.

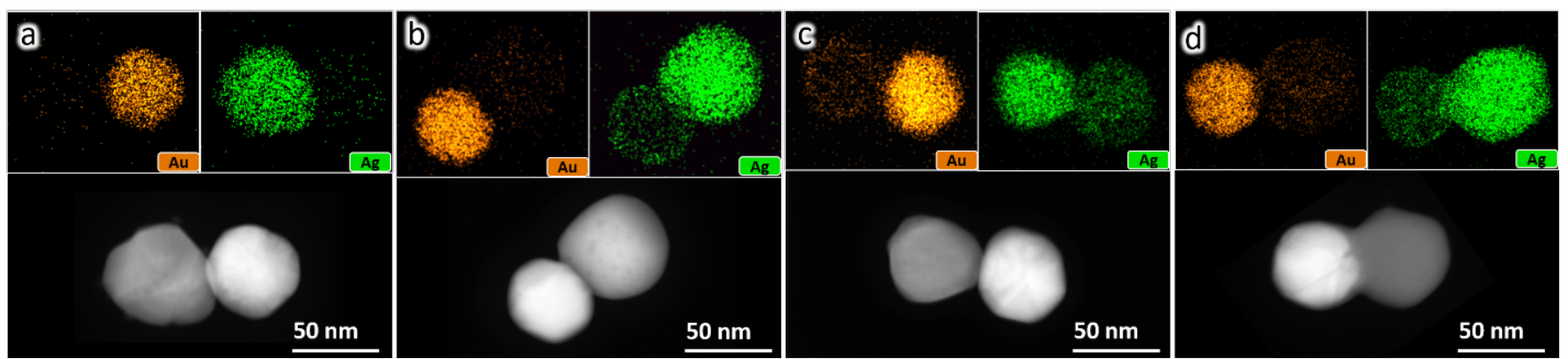

Figure 2. STEM images and EDX mappings of Ag-Au NPs after laser irradiation, showing four stages of Au-Ag NP welding. 